\title{
Single-Cell Proteomics Reveals Immune Persistence in Cutting-Edge CAR-T Therapies
}

\author{
IsoPlexis' single-cell functional phenotyping reveals key drivers that correlate with CAR-T cell \\ persistence in recent cancer studies.
}

The complete characterization of individual immune cells is critical in understanding the immune response to therapy. While the transcriptome is measured using RNA seq and the cell-surface phenotype via flow cytometry, complete cellular definition is incomplete without measuring the extracellular cytokines that are doing the functional work in cancer immunology.

The IsoPlexis technology can uniquely phenotype each immune cell by its extracellular function, termed "functional phenotyping." In many leading laboratories, IsoPlexis' proteomics hub, the IsoLight, and associated proteomics chips has become a standard tool to accelerate numerous immunotherapy programs.

CAR-T cell therapies have demonstrated many positive results. Patients who have shown a complete response to treatment have typically exhibited greater cell persistence and cell survival. For other patient cohorts, understanding how to maintain immune persistence remains a challenge. As demonstrated in two recent publications (discussed below), IsoPlexis' proteomics platform plays an important role in enabling researchers to predict which T cell therapies produce persistent results in difficult-to-treat situations, such as antigen escape.

\section{Polyfunctional CAR-T cells demonstrate superior anti-leukemic activity}

Fousek and colleagues headed by Hisham Abdel-Azim at Children's Hospital Los Angeles and Nabil Ahmed at Baylor College of Medicine recent published a report on CAR-T cells in Leukemia that nicely highlighted IsoPlexis' single-cell functional phenotyping. ${ }^{1}$

In pre-clinical settings for both solid and liquid tumors, combinatorial CAR-T cell therapies have demonstrated a clear advantage. Fousek et al. designed a combinatorial CAR-T cell for B-lineage acute lymphoblastic leukemia (BL-ALL). An estimated $39 \%$ of BL-ALL patients relapse after CD19-directed therapies.

The ubiquitously expressed CD19 is the usual target for BL-ALL; however, CD20 is expressed in about $50 \%$ of cases and CD22 is expressed in 80-90\%. In addition, CD20 is expressed by almost half of patients who have

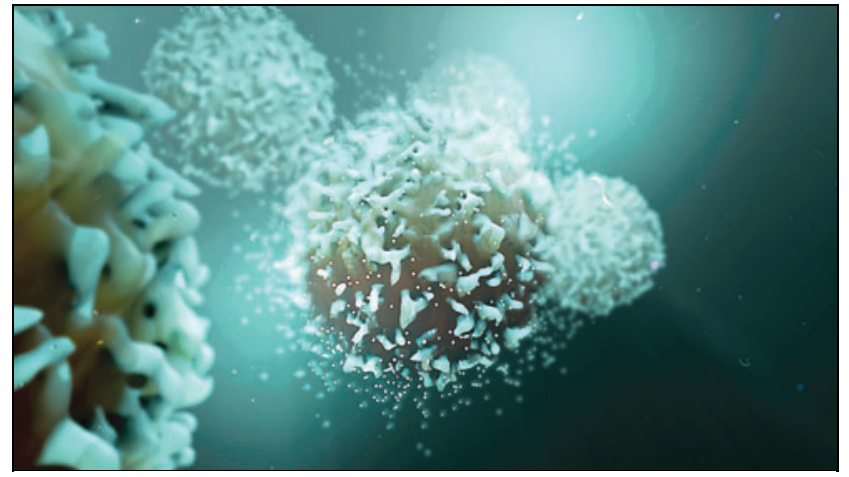

Credit: Design Cells.

mature B-lymphoid neoplasms, which are typically associated with a poor prognosis. It is also a predictor of relapse in adult patients when present at diagnosis. Antibody therapies targeting CD20 are now being used as front-line therapy for adults with BL-ALL. ${ }^{1}$

The authors reasoned that broadening the spectrum of CD19 CAR-T cells to include both CD20 and CD22 to target multiple antigens could be used as initial, secondary, or salvage therapies. They designed a CD19/ 20/22-targeting CAR-T cell by co-expressing individual CAR molecules on a single T cell. ${ }^{1}$

The IsoPlexis platform was used to characterize the novel cell therapy product for effectiveness and potency. Single-cell functional proteomics revealed the enhanced polyfunctional profiles of CD19/20/22 CAR$T$ cells with robust anti-tumor activity in response to CD19+ and CD19- tumor cells, supporting a potential advantage of this novel immunotherapy for patients with recalcitrant disease.

Importantly, IsoPlexis' Single-Cell Adaptive Immune solution demonstrated a marked upregulation of antigen-specific polyfunctionality in CD19/20/22 CAR-T cells compared to CD19 CAR-T cells. Polyfunctional CD19/20/22 CAR-T cells exhibited a greater formation of a functional chimeric antigen receptor immune synapse (CARIS) with both CD19- and CD19+ tumor cells, which could enhance their immunoactivity and prolong tumor-killing ability.

When this novel CAR-T product was tested in a long-term killing assay, the polyfunctional CD19/20/22 CAR-T cells 
eliminated target cells more promptly, sustained over five days of testing. ${ }^{1}$ This combination $\mathrm{T}$ cell therapy also effectively targeted and demonstrated superior anti-leukemic activity in vivo against CD19-escape BL-ALL and CD19+ BL-ALL.

The construct was found to be more activated when encountering target antigens (CD19+, CD20+, CD22) when compared with CD19 CAR-T cells. Only CD19/ 20/22 CAR-T cells maintained CD20 and CD22 CAR expression and consequently their activity against the target molecules.

\section{Polyfunctional CAR-T cells demonstrate immune persistence in solid tumors}

The IsoPlexis single-cell functional phenotyping technology was also highlighted in a recent high-impact study by Li and colleagues in the journal Gastroenterology. ${ }^{2}$ The research was led by Mitchell $\mathrm{Ho}$ at the National Cancer Institute.

While CAR-T therapies have shown success in treating $B$ cell malignancies, treating solid tumors using this approach remains challenging. The cells in blood malignancies are "approachable," whereas in a solid tumor microenvironment both reaching the target and maintaining persistence of the immunotherapy is difficult.

Glypican-3 (GPC3) is an oncofetal antigen involved in Wnt-dependent cell proliferation and is highly expressed in over $70 \%$ of hepatocellular carcinoma (HCC) and other solid tumors. As this protein can promote tumor growth, cellular therapies, such as CAR-T cells and bispecific antibodies, are targeting GPC $3 .^{2}$

$\mathrm{Li}$ et al. hypothesized that the function of CARs is affected by their epitope specificity, affinity, and functional activity. They sought to improve the anti-tumor activity with further modifications of a CAR construct, using the proteomically-barcoded single-cell technology to functionally characterize the experimental CAR-T designs.

As previous research had indicated a modest result in targeting GPC3, the researchers created CAR-T cells, based on the hYP7 and HN3 antibodies, which have high affinities for the C-lobe and N-lobe of GPC3, respectively, and performed preclinical tests in NOD/ SCID/IL-2Rgc null (NSG) mice. The modified CAR constructs also co-expressed a soluble PD-1 protein. $^{2}$

Mice injected with GPC3-specific humanized YP7 (hYP7) CAR-T cells had persistent expansion of T cells and subsets of polyfunctional CAR-T cells via antigen-induced selection. These T cells were observed in the tumor microenvironment and spleen for up to seven weeks after administration. The study demonstrated a positive correlation of enhanced polyfunctionality by single-cell functional proteomics with persistence of $\mathrm{T}$ cell response and in vivo killing capacity.

Importantly, this antigen-driven persistence and expansion of polyfunctional hYP7 CAR-T cells in the tumor microenvironment caused tumor regression in the HCC mouse model. Single-cell functional phenotyping uncovered the biological drivers and revealed the superior anti-tumor mechanisms of GPC3-targeted hYP7 CAR-T cell products in inhibiting Wnt signaling and promoting perforin/granzyme-mediated apoptosis in tumor cells through enhanced polyfunctional $\mathrm{CD} 8^{+} \mathrm{T}$ cell subsets.

The hYP7 CAR-T cells demonstrated a remarkably higher polyfunctional strength index (PSI) than the HN3 CAR-T cells when stimulated with either Hep3B or G1 cells. ${ }^{2}$ Polyfunctional T cells, a subset of T cells capable of co-producing two or more cytokines/chemokines at the single-cell level, have been associated with longterm immune responses in the clinical setting.

Furthermore, CD8 ${ }^{+}$hYP7 CAR-T cells had a PSI 8 to 11 times higher, thus more polyfunctional, than the $C D 4^{+}$ hYP7 CAR-T cells co-cultured with Hep3B and G1 cells. The researchers also discovered that HCC patientderived polyfunctional CD8 ${ }^{+}$hYP7 CAR-T cells contained effector cytokines (granzyme B, IFN- $\gamma$, and perforin) as well as cytokines not secreted by CD8 ${ }^{+}$hYP7 CAR-T cells from healthy donors (TNF- $\alpha$, MIP- $1 \beta$, and IL- 8 ).

Sixty-six percent of mice treated with the hYP7 CAR-T cells had their tumors eliminated by week 3, while mice treated with the HN3 CAR-T cells showed no reduction in tumor burden. The hYP7 CAR-T treated mice also continued to be free of tumors after additional Hep3B cells were introduced. The authors hypothesized that differences in persistence, integration site preferences, and T cell polyfunctionality may contribute to the discrepancy between the efficacies of the CAR constructs. ${ }^{2}$

\section{Conclusion}

IsoPlexis' single-cell functional phenotyping has revealed critical functional drivers that correlated with CAR-T cell persistence in both BL-ALL and HCC indications. This demonstrates how early information on T cell polyfunctional capacity provides clear value to facilitate decisions on lead candidates in the development of cell therapies.

\section{References}

1. Fousek K, Watanabe J, Joseph S, et al. CAR T-cells that target acute B-lineage leukemia irrespective of CD19 expression. Leukemia (2020). doi.org/10.1038/s41375-020-0792-2

2. Li D, Li N, Zhang YF, et al. Persistent Polyfunctional Chimeric Antigen Receptor T Cells That Target Glypican 3 Eliminate Orthotopic Hepatocellular Carcinomas in Mice. Gastroenterology 2020 Feb 11. pii: S0016-5085(20)30211-0. doi: 10.1053/j.gastro.2020.02.011. 